AperTO - Archivio Istituzionale Open Access dell'Università di Torino

\title{
Small sample bias in MSM estimation of agent-based models
}

\section{This is the author's manuscript}

Original Citation:

Availability:

This version is available http://hdl.handle.net/2318/108108

since

Publisher:

Springer

Terms of use:

Open Access

Anyone can freely access the full text of works made available as "Open Access". Works made available under a Creative Commons license can be used according to the terms and conditions of said license. Use of all other works requires consent of the right holder (author or publisher) if not exempted from copyright protection by the applicable law. 


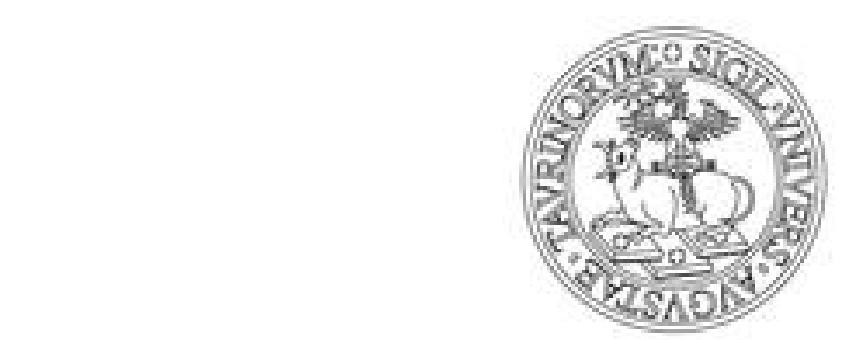

\section{UNIVERSITÀ DEGLI STUDI DI TORINO}

This is an author version of the contribution published on:

Jakob Grazzini, Matteo Guido Richiardi, Lisa Sella

Small sample bias in MSM estimation of agent-based models

Editor: Springer

2012

ISBN: 9783642313004

in

Andrea Teglio, Simone Alfarano, Eva Camacho-Cuena, Miguel Gines-Vilar

Managing Market Complexity. The Approach of Artificial Economics $237-245$ 


\title{
Small sample bias in MSM estimation of agent-based models
}

\author{
Jakob Grazzini, Matteo Richiardi and Lisa Sella
}

\begin{abstract}
Starting from an agent-based interpretation of the well-known Bass innovation diffusion model, we perform a Montecarlo analysis of the performance of a method of simulated moment (MSM) estimator. We show that nonlinearities of the moments lead to a small bias in the estimates in small populations, although our estimates are consistent and converge to the true values as population size increases. Our approach can be generalized to the estimation of more complex agent-based models.
\end{abstract}

\section{Introduction}

In this chapter we present an example of the use of simulation-based econometric techniques for the estimation of agent-based (AB) models. While the full details of the estimation strategy can be found in [18], here we focus on the small sample properties of the simulated moment estimator. We show the existence of a small sample bias in the estimates, which however vanishes as the sample size increases. The bias turns out to be originated by non-linearity of the moments selected for estimation, a feature that is quite common in $\mathrm{AB}$ models as non-linearities are intrinsically linked to complex systems. As an application, we use a discrete-time operationalization of the well-known Bass model of innovation diffusion [4]. This model describes the evolution over time of the number of adopters by means of a differential equation. We reinterpret this equation as an individual probability of adoption, which depends on the number of linked agents that have already adopted.

Jakob Grazzini

Catholic University of Milan, Institute of Economic Theory and Quantitative Methods, via Necchi 5, 20123 Milano, e-mail: jakobgrazzini@gmail.com

Matteo Richiardi

University of Turin, Department of Economics and LABORatorio Revelli, Collegio Carlo Alberto, via Real Collegio 30, 10024 Moncalieri, Torino e-mail: matteo.richiardi@unito.it

Lisa Sella

Ceris - CNR, via Real Collegio 30, 10024 Moncalieri, Torino e-mail: lisa.sella@unito.it 
Our work contributes to the still sparse literature on the structural estimation of $\mathrm{AB}$ models. Indeed, this is identified as a key weakness of $\mathrm{AB}$ models: $\mathrm{AB}$ models often remain at a theoretical level and lack a sound empirical grounding [13]. When present, this is often limited to some ad-hoc calibration of the relevant parameters. However, estimation is crucial for the empirical validation of the model, for comparing the model with other available models, and for policy analysis.

The main reason for this state of affairs is that, even if $\mathrm{AB}$ models can be regarded as a set of mathematical equations [26], their properties remain hidden in the complexity of the relations among the many elements in the model. The lack of an analytical formalization linking the behavior of the agents with the outcome of the system impedes a traditional approach to model estimation, and calls for computational methods. These methods, known as simulation-based estimation techniques $[38,37]$, have been originally developed in the econometric literature to deal with analytical models leading to criterion functions without simple analytical expression (for instance because of integrals of large dimensions in the probability density function or in the moments). Their application to $\mathrm{AB}$ models, however, is not straightforward. Consequently, only a handful of examples exist on the structural estimation of $\mathrm{AB}$ models. [39] and [15] estimate respectively 2 and 3 parameters of an $\mathrm{AB}$ model of the foreign exchange market introduced by [22, 23], by employing the method of simulated moments (MSM). Their focus is on optimization heuristics. In [40] they deal with the problem of moments selection, and propose a set of statistics on exchange rate returns to estimate models of exchange rate. In [17] the consistency of the MSM estimator applied to agent-based models is investigated.

The MSM is only one among the many simulation based econometric techniques that can be used, but it is relatively simple and intuitive and therefore it gained popularity in the $\mathrm{AB}$ modelling community. ${ }^{1}$ However, it is still considered by many more or less as a black box. By means of Montecarlo experiments on our illustrative model, we aim at opening up this black box.

The chapter is structured as follows. Section 2 describes the original Bass model. Section 3 describes our AB version of the Bass model. Section 4 gives a brief overview of the estimation strategy, which is explained in more details in our companion paper. Section 5 focuses on the small sample properties of the estimators, and describes the origins of the bias. Section 6 concludes.

\footnotetext{
${ }^{1}$ The use of other techniques is even more limited. [6] estimate, by means of a non-linear least square method, a dynamic asset pricing model characterized by agents with heterogeneous beliefs. [9] use a Gaussian Process emulator of scalar computer model output for sensitivity analysis, (Bayesian) calibration, and model comparison. Their methodology is relevant for models that are expensive to run, in money or time, and for which the number of possible evaluations is therefore limited. Finally, $[3,2]$ estimate $\mathrm{AB}$ models that are simple enough to derive a closed form solution for the distribution of relevant statistics.
} 


\section{The Bass model}

The Bass model [4], which provides a mathematical explanation of the different stages of product adoption described in the seminal work by Everett Rogers [34] (innovators, early adopters, early majority, large majority, and laggards), and formalizes the crucial distinction between innovators and imitators, is considered as one of the most important empirical generalization in marketing, and it is widely used in sales and technology adoption analysis.

The model is an example of early epidemic models of innovation diffusion [14]. It consists of a differential equation that specifies the rate of adoption $h(t)$ as a function of an external force and an internal (endogenous) one. The external influence is constant over time and represents the effects of advertisement, while the internal influence depends on how many others have already adopted at time $t$ and formalizes word-of-mouth:

$$
h(t)=p+q F(t)
$$

where $F(t)=N(t) / m$ is the c.d.f. of adopters, that is the ratio of those who have already adopted $(N(t))$ over the number of potential adopters (the market potential $m) . p$ is the parameter for external influence and $q$ is the parameter for internal influence, with $p+q<1$. $^{2}$

The internal influence in the Bass model operates as a mean field force over the whole population of potential adopters: every individual is connected to every other individual in the population. At the beginning the adoption is slow since the number of agents that have already adopted is small and therefore the interaction term is negligible. Once the number of adopters starts to increase, the probability of adoption for those who have not already adopted (the population at risk) increases and the diffusion gets faster. As the population at risk gets smaller, the number of new adopters decreases until the diffusion process is completed. The diffusion dynamic follows a typical S-curve.

The model is deterministic and thus requires some sort of adaptation to be taken to the data. The literature on the estimation of the Bass model has followed two strategies. The most popular is to add a noise to the aggregate pattern of adoptions predicted by the model $[4,36,21,6]$. We call this approach the macro approach. The noise is meant to catch not only sampling variability and measurement errors, but also specification errors. The properties of the noise determine the properties of the estimators. What is most important here, however, is that the estimators that have been proposed following this approach are not even consistent, given that (i) convergence cannot be obtained by letting the observation period grow, because the process is finite and saturation (that is, full adoption) is sooner or later obtained, and (ii) convergence cannot be obtained neither by letting population size grow, because

\footnotetext{
2 This specification of the hazard function had already been introduced to characterize innovation diffusion processes prior to Bass' work $[8,30]$. However, empirical applications were scant, because knowledge of the number of potential (and ultimate) adopters $m$ was required to compute $F(t)$. Bass contribution was to express the adoption ceiling as a parameter, which could be estimated together with $p$ and $q$ using aggregate sales data.
} 
the noise is added directly to the aggregate outcome. A second strategy is to consider the adoption process as a duration model assuming equation 1 specifies an homogeneous hazard rate for all individuals in the population [35]. We call this approach the micro approach. In this case the only source of variability comes from sampling errors, while the model is assumed to be correctly specified. The corresponding ML estimator is consistent in population size.

\section{The $\mathrm{AB}$ version}

We identify two main shortcomings in the literature we have briefly reviewed above: the macro approach gives raise to inconsistent estimates, while the micro approach is not able to account for the discrete nature of many diffusion processes. We now elaborate on the latter issue.

Our model shares with the micro approach the same interpretation of equation 1 as an individual probability of adoption, conditional of being still at risk, but considers that adoption can take place only at discrete time intervals, rather than continuously. This is more appropriate for many applications (think for instance of movie attendance, where most individuals go the cinema on Saturday night and in any case not on a $24 / 7$ basis). Even when the process is indeed continuous, information on cumulative adoption generally becomes available only at discrete time intervals, which in our modelling framework makes the decision to adopt essentially discrete. In other words, the kind of mean-field interaction assumed in the Bass model requires that information is centrally collected and then diffused. Individuals have to rely on data collection by some statistical agency to take their decisions - exactly as the researcher does to analyze those decisions and estimate the parameters of the model. If data release coincides with the information release on which individuals take their decisions (which is quite plausible if the network structure is highly connected), a discrete framework is more appropriate. However, the micro approach assumes a continuous duration model. On the other hand, our estimation strategy is tailored to the discrete nature of the process.

\section{Estimation}

Let's consider an homogeneous population of $m$ individuals, where the individual hazard of adoption is given by eq. 1. As standard in this literature, we assume that the individuals act independently of each other within each time interval. In [18] we develop estimators for $p$ and $q$ as a function of $m$, and show that these estimators are unbiased, consistent and asymptotically normal for large populations $m$. We then propose a MSM estimator $[31,33,24,11]$ to estimate the market potential $m$, which minimize the distance between the observed moment $\tau_{r}$ (which is given) and the simulated moment $\tau_{s}(m)$, obtained by simulating the adoption time of $m$ individuals 
with $h_{t}=\hat{p}(m)+\hat{q}(m) N_{t-1} / m$. The moment we use is the mean adoption time for those who have adopted in the observation period:

$$
\tau(T, m)=\frac{1}{N_{t}} \sum_{t=0}^{T}\left(t n_{t}\right)
$$

Figure 1 shows how the moment responds to changes in $m$, for fixed values of the other parameters.

For each value of $m, \tau(T, m)$ is a random variable. Figure 2, which depicts its skewness, shows that it is not significantly different from 0 . The distribution is therefore (almost) symmetric, a property that will turn out to be important in understanding the direction of the small sample bias.

In facts, our final estimators for $m, p$ and $q$ are consistent but subject to a small sample bias, although not large. Preliminary findings show that, if the process is indeed discrete, they perform very well with respect to the other estimators proposed in the literature, which also suffer from small sample bias. ${ }^{3}$

\section{Small sample bias}

Where does the small sample bias come from? Figure 1 contains the answer. The theoretical moment is not linear in $m$. If the observed moment, which is a random variable, is symmetric and centered around the theoretical moment, we have

$$
E\left[\tau^{-1}(m) \neq m\right]
$$

The direction of the bias depends on the sign of the first and second derivatives of the moment, at the true value of the parameter (see also [17]). For example, if the first derivative is positive, a positive second derivative implies that the moment is accelerating in $m$ : it is less steep at the left than at the right of the true value of the parameter. Therefore, a low realization of the moment $\tau_{L}$ leads to a very low inferred value of the parameter $\hat{m}_{L}=\tau^{-1}\left(\tau_{L}\right)$, while a high realization $\tau_{H}$ leads to a not-so-high inferred value $\hat{m}_{H}=\tau^{-1}\left(\tau_{H}\right)$, with $E\left[\hat{m}_{L}, \hat{m}_{H}\right]<m$. We get a downward bias. Figure 3 illustrates the possible cases.

Given the shape of the mean adoption time for the adopters (figure 1), an upward bias is expected for $\hat{m}$ in small samples, that is exactly what we get from the Montecarlo analysis. The bias in the other parameters is consequential: an upward bias in $\hat{m}$ implies a downward bias in $\hat{p}$ and $\hat{q}$, given that the simulated penetration rate $F(t)$ is lower than the true (but unobserved) one.

The bias vanishes as the population of potential adopters increases because with a higher number of adopters the uncertainty over their mean adoption time reduces: the mean adoption time converges to its theoretical value. Therefore, any extrac-

\footnotetext{
${ }^{3}$ Moreover, most estimators based on the macro representation of the diffusion process are not even consistent (see our companion paper [18] for a discussion).
} 
tion of the real data would produce the same mean adoption time, and the problem outlined above disappears.

\section{Conclusions}

In this paper we have shown an application of simulation-based econometric techniques to the estimation of $\mathrm{AB}$ models. The model chosen for the demonstration is important both because innovation diffusion is a wide area of application of $A B$ models [10] and because the model has been widely studied in its analytical form. However, the estimation strategies proposed in the literature have either poor properties, or are limited to the case of a continuous diffusion process. Conversely, our three-stage estimator assumes a discrete process, that converges to a continuous one as the frequency of the data increases. The estimator is consistent, but estimates in small samples are biased: in particular, whose of the market potential are upward biased, while those of the influence parameters are downward biased. This happens also with the consistent estimator proposed in the literature for the continuous case, and is due to non-linearities in the model. In our case, the bias could in principle be solved by knowing the analytical expression of the conditional moment, but this is typically beyond reach in an $\mathrm{AB}$ model. However, the bias could be reduced by applying a monotonic transformation of the moments used for estimation, in order to linearize them. In [18] we show that the bias is anyhow quite small; therefore, it should not be considered as a major problem in this application. It is however illustrative of a problem that $\mathrm{AB}$ modellers interested in the empirical validation of their models should be aware of.

Finally, note that our estimation strategy has been carried out in the simple case of fully connected network, but it can be seen as a first step toward the estimation of diffusion model with more realistic network structures. Future research should then investigate to what extent these richer network structures can be estimated from aggregate diffusion data.

\section{Acknowledgements}

We thanks Sebastiano Alessio Del Re, with whom we originally discussed the project and who developed the initial Matlab code. Preliminary versions of the paper were presented at the 37th and 38th Annual Meeting of the Eastern Economic Association, respectively in New York (2011) and Boston (2012) and at the GSDP Agent-based modelling workshop in Paris, September 8-10 2011. We thanks the participants to those events for their comments. Jakob Grazzini acknowledges the financial support from the European Union, Seventh Framework Programme FP7/2007-2013 under grant agreement no. CRISIS-ICT-2011-288501. 


\section{References}

1. Van den Bulte, C. and Lilien, G.L. (1997) Bias and Systematic Change in the Parameter Estimates of Macro-Level Diffusion Models. Marketing Science 16(4), 338-353.

2. Alfarano, S. and Wagner, T. and Lux, F. (2006) Estimation of a Simple Agent-Based Model of Financial Markets: An Application to Australian Stock and Foreign Exchange Data. Physica A $370(1), 38-42$

3. Alfarano, S. and Wagner, T. and Lux, F. (2005) Estimation of Agent-Based Models: The Case of an Asymmetric Herding Model. Computational Economics 26, 19-49

4. Bass, F.M.(1969) A New Product Growth for Model Consumer Durables. Management Science $15,215-227$

5. Bonabeau, E. (2002) Agent-based modeling: Methods and techniques for simulating human systems. PNAS 99(3), 7280-7287

6. Boswick, H.P. and Hommes, C.H. and Manzan S. (2007) Behavioral Heterogeneity in Stock Prices. Journal of Economic Dynamics and Control 31(6), 1938-1970

7. Boswijk, H.P. and Franses, P.H. (2005) On the Econometrics of the Bass Diffusion Model. Journal of Business and Economic Statistics 23(3), 255-268

8. Coleman, J.S. (1964) Introduction to mathematical sociology.The Free Press of Glencoe, London

9. Dancik, G.M. and Jones, D.E. and Dorman, K.S. (2010) Parameter estimation and sensitivity analysis in an agent-based model of Leishmania major infection. J Theor Biol. 262(3), 398412

10. Dawid, H. (2006) Agent-Based Models of Innovation and Technological Change. In: Handbook of Computational Economics, Vol. 2: Agent-Based Computational Economics, NorthHolland/Elsevier, Amsterdam

11. Duffie, D. and Singleton, K.J. (1993) Simulated moments estimation of Markov models of asset prices. Econometrica 61, 929-952

12. Gallant, A.R. and Tauchen, G. (1996) Which Moments to Match?. Econometric Theory 12, 657-681

13. Gallegati, M. and Richiardi, M. (2009) Agent-based Modelling in Economics and Complexity. In: Meyer R.A. (eds) Encyclopedia of Complexity and System Science, Springer

14. Geroski, P. A. (2000) Models of technology diffusion. Research Policy 29(4-5), 603-625

15. Gilli, M. and Winker, P. (2003) A global optimization heuristic for estimating agent based models. Computational Statistics and Data Analysis 42(2) 299-312

16. Gourieroux, C. and Monfort A. (1996) Simulation-Based Econometric Methods, Oxford University Press, New York

17. Grazzini, J. (2011) Estimating Micromotives from Macrobehavior. University of Turin Department of Economics Working Papers Series 2011(11)

18. Grazzini J. Richiardi M.G. and Sella L. (2012) Indirect estimation of agent-based models. An application to a simple diffusion model. Complexity Economics 1(2), forthcoming

19. Hendry, D.F. (2002) Model Identification and Non-Unique Structure. University of Oxford Economics Papers Series 2002-W10

20. Ivanov, A.V. (1997) Asymptotic Theory of Nonlinear Regression, Kluwer, Dordrecht

21. Jain, D.C. and Rao, R.C. (1990) Effect of Price on the Demand for Durables: Modeling, Estimation, and Findings. Journal of Business and Economic Statistics (8, 163-170

22. Kirman, A. (1991) Epidemics of opinion and speculative bubbles in financial markets. In: Taylor, M. (eds) Money and Financial Markets, Macmillan

23. Kirman, A. (1993) Ants, rationality, and recruitment. The Quarterly Journal of Economics $108,137-156$

24. B. Lee and B. Ingram (1991) Simulation estimation of time series models. Journal of Econometrics 47, 197-205

25. Peter J. Lenk and Ambar G. Rao (1990) New Models from Old: Forecasting Product Adoption by Hierarchical Bayes Procedures, Marketing Science 9(1), 42-53 
26. Roberto Leombruni and Matteo Richiardi (2005) Why are economists sceptical about agentbased simulations? Physica A 3559, 103-109

27. Liu, T.C. (1960) Underidentification, Structural Estimation, and Forecasting. Econometrica $28,855-865$

28. Mahajan, V. and Muller, E. and Bass, F. (1995) Diffusion of new products: Empirical generalizations and managerial uses. Marketing Science 14(3)

29. Mahajan, V. and Muller, E. and Bass, F. (1990) New Product Diffusion Models in Marketing: A Review and Directions for Research. Journal of Marketing 54(1), 1-2

30. Mansfield, E. (1961) Technical Change and the Rate of Imitation. Econometrica 29, 741-766

31. McFadden, D. (1989) A Method of Simulated Moments for Estimation of Discrete Response Models Without Numerical Integration. Econometrica 57, 995-1026

32. Miller J.H. and Page S.E. (2006) Complex Adaptive Systems: An Introduction to Computational Models of Social Life. Princeton University Press, Princeton, NY

33. Pakes, A. and Pollard, D. (1989) Simulation and the Asymptotics of Optimization Estimators. Econometrica 57, 1027-1057

34. Rogers, E. M. (1962) Diffusion of innovations. Free Press, New York

35. Schmittlein D.C. and Mahajan V. (1982) Maximum Likelihood Estimation for an Innovation Diffusion Model of New Product Acceptance. Marketing Science 1(1) 57-78

36. Srinivasan, V. and Mason, C.H (1986) Nonlinear Least Square Estimation of New Product Diffusion Models. Marketing Science 5(2), 169-178

37. Stern S. (2000) Simulation-based inference in econometrics: motivation and methods. In: Mariano, R. Schuermann, T. and Weeks M.J. (eds) Simulation-based inference in econometrics: methods and applications, Cambridge University Press

38. Stern S. (1997) Simulation Based Estimation. Journal of Economic Literature 35(4) 20062039

39. Winker, P. and Gilli, M. (2001) Indirect estimation of the parameters of agent based models of financial markets. School of Business Administration, International University in Germany $03 / 2001$

40. Winker P., Gilli M. and Jeleskovic V. (2007) An objective function for simulation based inference on exchange rate data. Journal of Economic Interaction and Coordination 2, 125-145

41. L. Tesfatsion and K.L. Judd (2006) Handbook of Computational Economics, Volume 2: Agent-Based Computational Economics, North-Holland 


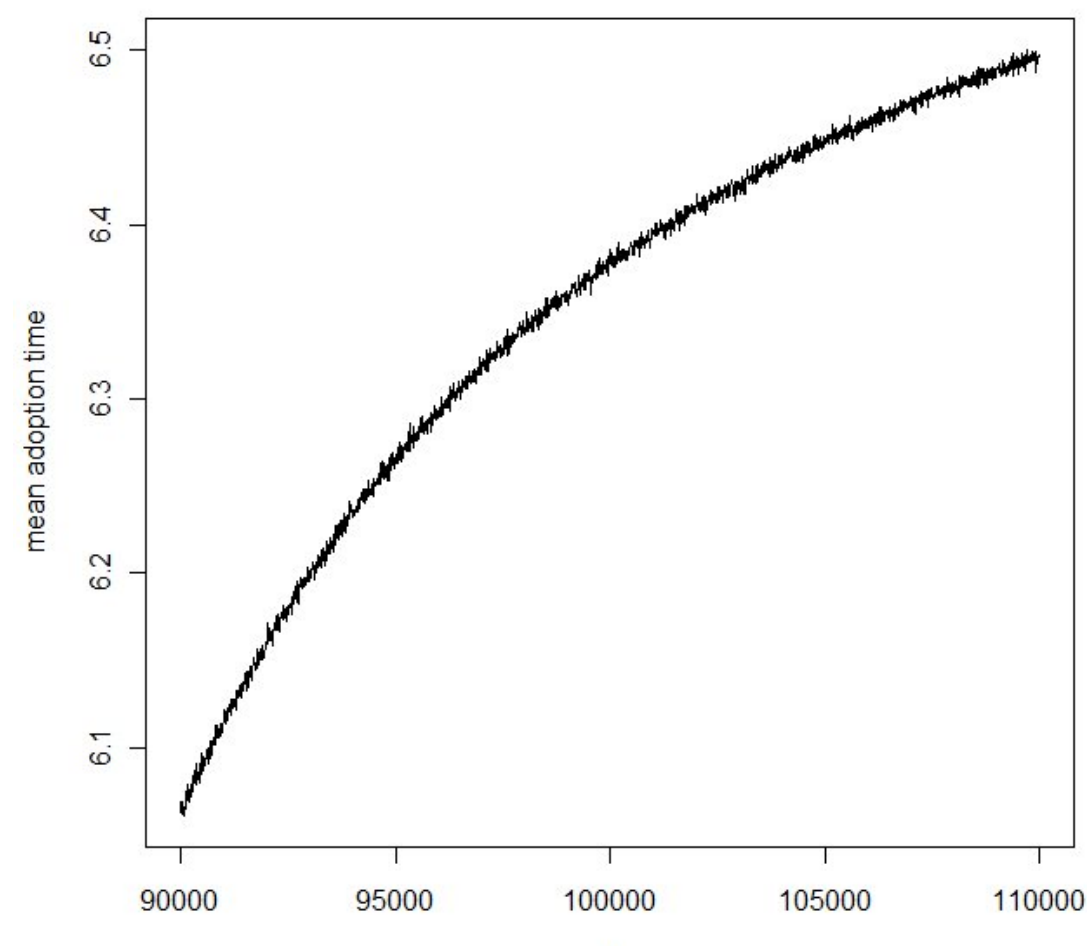

$\mathrm{m}$

Fig. 1: Average adoption time for the adopters $(\tau(T, m))$, different values of $m$. Other parameters: $p=0.03, q=0.4, T=10$. Ten artificial adoption sequences are simulated for each value of $m$. For each sequence, 10 replications of the estimation procedure are performed, with different pseudorandom numbers. For each set of estimated parameters, $\tau(T, m)$ is computed. The graph reports average values. 


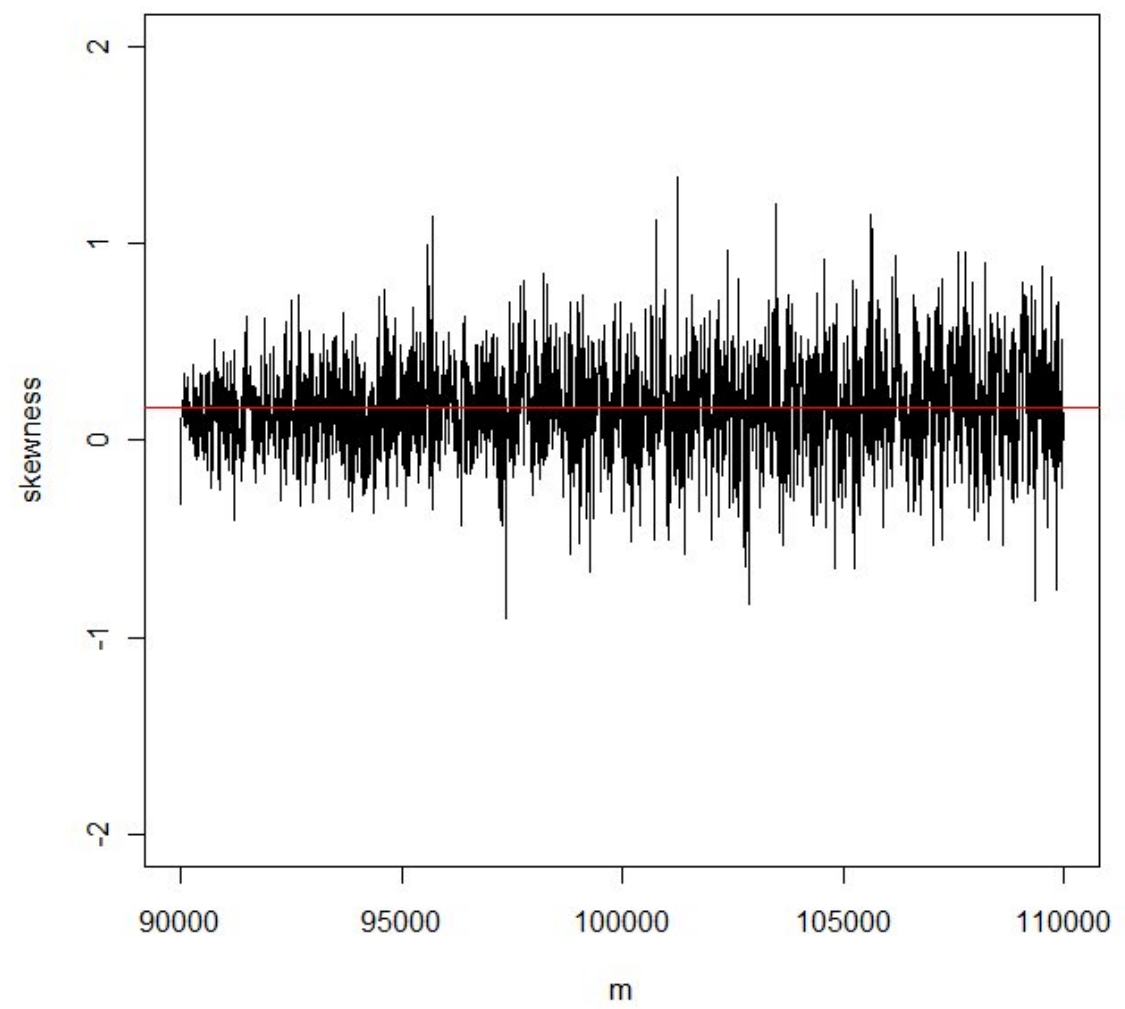

Fig. 2: Skewness of the $\tau(T, m)$ distribution, different values of $m$. Other parameters: $p=0.03, q=$ $0.4, T=10$. Ten artificial adoption sequences are simulated for each value of $m$. For each sequence, 10 replications of the estimation procedure are performed, with different pseudo-random numbers. For each set of estimated parameters, $\tau(T, m)$ is computed. The graph reports the skewness of the conditional distributions $\tau(T, m \mid m)$. 


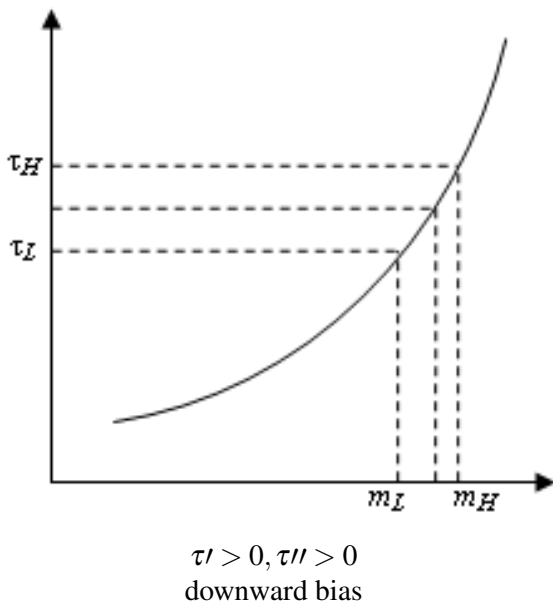

downward bias

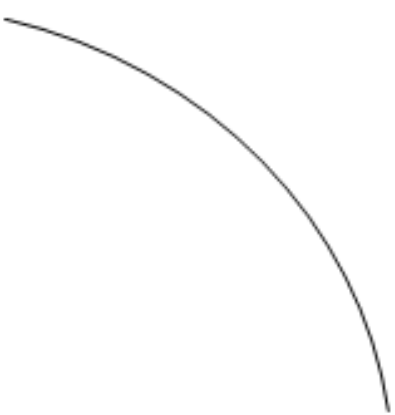

$\tau \prime<0, \tau \prime \prime<0$

downward bias

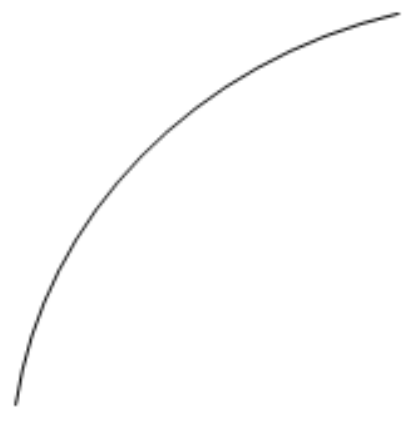

$\tau \prime>0, \tau \prime \prime<0$

upward bias

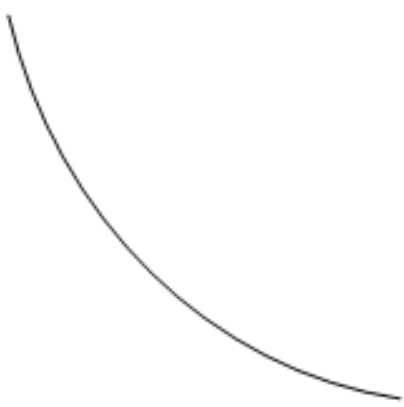

$\tau \prime<0, \tau \prime \prime>0$

upward bias

Fig. 3: Distribution of estimated coefficients. True values of the parameters: $p=0.03, q=0.4, m=$ $1,000,000$. The estimates are based on observations on the first $T=10$ periods. 